\title{
Unveiling the fundamental properties of Gamma-Ray Burst host galaxies
}

\author{
Sandra Savaglio \\ Max Planck Institute for extraterrestrial Physics, \\ Giessenbachstr., 85741, Garching, Germany \\ email: savaglio@mpe.mpg.de
}

\begin{abstract}
The galaxies hosting the most energetic explosions in the universe, the gammaray bursts (GRBs), are generally found to be low-mass, metal-poor, blue and star forming. However, the majority of the targets investigated so far (less than 100) are at relatively low redshift, $z<2$. We know that at low redshift, the cosmic star formation is predominantly in small galaxies. Therefore, at low redshift, long-duration GRBs, which are associated with massive stars, are expected to be in small galaxies. Preliminary investigations of the stellar mass function of $z<1.5$ GRB hosts does not indicate that these galaxies are different from the general population of nearby star-forming galaxies. At high- $z$, it is still unclear whether GRB hosts are different. Recent results indicate that a fraction of them might be in dusty regions of massive galaxies. Remarkable is the a super-solar metallicity measured in the interstellar medium of a $z=3.57$ GRB host.
\end{abstract}

Keywords. Gamma rays: bursts, observations, ISM: abundances, galaxies: evolution

\section{Introduction}

GRB host galaxies are traditionally thought to be associated with small, metal- and dust-poor, star forming galaxies. However, most of these galaxies studied in detail are at redshift smaller than $z=1.5$. We still do not know whether the situation at higher redshift is different. We know with high confidence that the progenitor of most GRBs, the long-duration ones, is a massive and short-lived star $\left(M>30 \mathrm{M}_{\odot}\right.$; Heger et al. 2003). Therefore, their hosts are likely star forming galaxies. The star-forming galaxy population experienced major changes in the global history of the universe, for instance, for the star-formation rate (SFR), the galaxy stellar mass, and the merger rate.

The cosmic star-formation rate dropped by a factor of 50 from $z \sim 1.8$ (Hayes et al. 2010 ), and it transited from large galaxies in the past to small galaxies today (Juneau et al. 2005). The evolution of the fraction of major mergers of massive galaxies in the same time interval dropped by a factor of $\sim 25$ (Bluck et al. 2012). The assembly growth of galaxy stellar mass shows that the mass density increased by only a factor of $\sim 50 \%$ since $z=1$ (Sobral et al. 2012). The mass-metallicity relation in the local universe, and its redshift evolution, show that large galaxies reached high metallicities early on, while small galaxies are chemically more slow (Savaglio et al. 2005). These relations are affected by the galaxy SFR, such that, for a given stellar mass, metallicities tend to be lower for higher SFRs (Mannucci et al. 2010).

Therefore, if from one hand it is not surprising that, in the local universe, most GRBs occur in small star-forming and metal-poor galaxies, at high redshift, more massive galaxies, active and metal-rich galaxies might have hosted a large fraction of the events. Numerous new and deeper observations start to suggest that the canonical view might be affected by a combination of the difficulty of detecting distant targets (Krühler et al. 


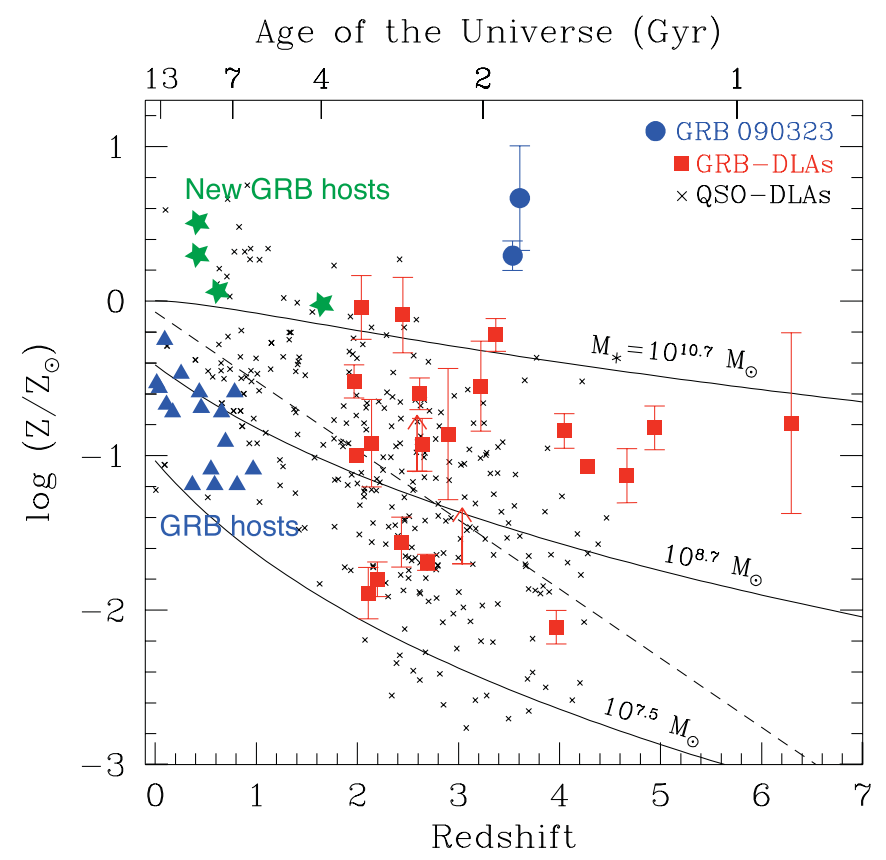

Figure 1. Metallicity as a function of redshift in galaxies. Metallicities in the two absorbers detected in GRB 090323 (blue dots) are from Savaglio et al. (2012). Red squares are metallicities of other GRB-DLAs. Blue triangles are GRB host metallicities measured from emission lines at low redshift (Savaglio et al. 2009). More recent metal determinations show high metallicities (Levesque et al. 2010; Perley et al. 2011; Krühler et al. 2012; Niino et al. 2012). Metallicities in other high- $z$ absorbers detected in QSO spectra (QSO-DLAs) are black crosses. The dashed line is the linear correlation for QSO-DLA points. Solid curves are average metallicities expected for star-forming galaxies with different stellar masses (Savaglio et al. 2005).

2011) and the redshift evolution of galaxy fundamental parameters. The peculiar strong double absorption system recently studied in the spectrum of the distant GRB 090323 $(z=3.57)$, and the measured super-solar metallicity (Fig. 1) supports the same idea (Savaglio et al. 2012). One of the next major goals is to study the stellar-mass function of galaxies hosting GRBs.

\section{The stellar mass of GRB hosts}

The investigation of the mass function (MF) of GRB hosts is vaguely possible, if at all, at low redshift only, due to the small number statistics. The hosting galaxy is studied in only half of all GRBs with known redshift (more than 240), mainly at $z<2.3$ (87 galaxies, $71 \%$ of the total). The galaxy MF is a fundamental mean through which the cosmic change of galaxies can be identified. Widely investigated in the local universe (e.g., Baldry et al. 2008), and at high redshift (Santini et al. 2011), it shows that small galaxies are the most common ones at $z=0$, and even more so at $z>2$.

The MF was never derived for galaxies hosting GRBs, due to the small number statistics and the difficulty in defining the sample completeness. Nevertheless, the identification of GRB hosts, which is, to a first-order approximation, independent of the galaxy brightness, makes the investigation of the $z>0 \mathrm{MF}$ in the low stellar-mass regime $\left(M_{*}<10^{10}\right.$ $\left.\mathrm{M}_{\odot}\right)$ a possible task. That will help to establish whether these galaxies belong to a unique population, or naturally fill the low-mass end of the galaxy MF. The 45 GRB hosts studied by Savaglio et al. (2009) did not show evidence for deviation from normal galaxies. In 


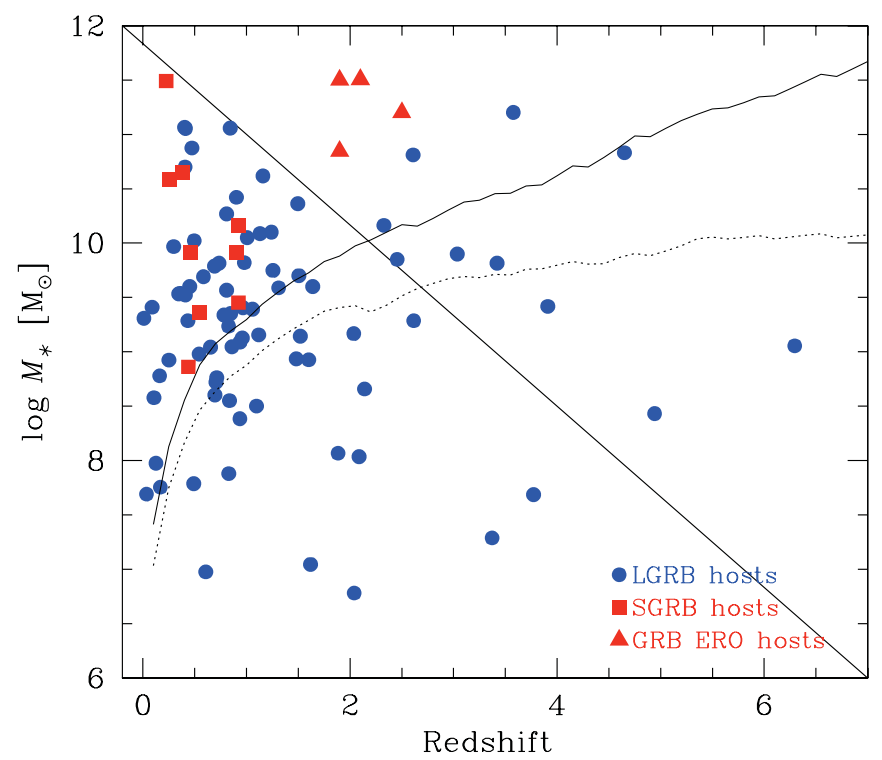

Figure 2. Stellar mass of GRB hosts as a function of redshift. Circles and squares are galaxies associated with long and short GRBs with spectroscopic redshift, respectively (Savaglio et al. in prep.). Triangles are galaxies with photometric redshifts associated with extremely red objects (see Hunt et al. 2011 for details). The solid and dashed lines represent the stellar mass as a function of redshift of a galaxy with $\mathrm{AB} K$-band magnitude $m_{K}=24.3$, and old stellar population or constant SFR, respectively.

our ongoing work (Savaglio et al., in prep.), the GRB host sample is more than 2.5 times larger. Fig. 2 displays the preliminary stellar mass as a function of redshift for 88 hosts with spectroscopic redshift, and 4 hosts associated with extremely red objects (EROs) with photometric redshift. A large fraction of GRB hosts are massive, in particular those associated with EROs (see Hunt et al. 2011, and details therein).

To investigate the GRB host MF, one needs to select the sample according to the volume limited criteria. This is the most critical part, relatively under control for a large sample. In any magnitude-limited galaxy sample, the mass distribution is dominated by massive galaxies. When one corrects for volume, then low-mass galaxies dominate. The sample selection for GRB hosts is very different. If $100 \%$ of detected GRBs were followed up and galaxy masses measured, then part of the relevant volume calculation would be the flux limit of the GRB-detecting telescopes (e.g., Swift) as a function of redshift.

In our preliminary study, we just investigated the shape of the MF, by normalizing it to that of field galaxies. The MF was estimated for 31 GRB hosts at $z<1.5$ and stellar mass $M_{*}>10^{9.25} \mathrm{M}_{\odot}$. Field galaxies in the local universe (Baldry et al. 2008) and star-forming galaxies at $z \sim 1$ (Pozzetti et al. 2010; Gilbank et al. 2011) indicate that the general shape of the MF did not change remarkably. Today we have $\sim 3$ times more galaxies with $M_{*}>3 \times 10^{8} \mathrm{M}_{\odot}$ than back then. The GRB-host MF shows a similar shape. GRB hosts have a reputation of being small star-forming galaxies, but this is not apparent from their relatively flat $\mathrm{MF}$.

The most important issue to solve now is the primary selection function of Swift in a luminosity vs. redshift plot. We will have to determine whether the actual luminosity distribution of GRBs (in $\gamma$-rays) is a gaussian, a power law, or a Schechter luminosity function. Once this is under control, we can apply the volume-limited calculation. 


\section{Conclusion}

The importance of investigating galaxies hosting GRBs has become evident because of their connection with the most active, observationally hostile, and remote regions of the universe. Recent works have shown that some (long) GRB hosts are metal-rich, massive, and dusty, with high star-formation rates, in contrast with the low-metallicity, low-mass hosts commonly found at $z<1.5$. The impact on cosmology is still limited by the small number statistics. One important step forward is the multi-wavelength (from X-ray to radio) approach, which can statistically quantify the importance of red galaxies associated with dark GRBs. Exploitation of the long wavelength regime is now possible thanks to the capabilities of new ground-based telescopes (SCUBA-2, APEX, ALMA, ATCA) and space missions (Spitzer, Herschel, WISE). This will ultimately establish whether some high- $z$ GRB hosts are connected to dusty sub-millimeter galaxies.

\section{Acknowledgement}

The author is particularly indebted to S. Basa, J. Greiner, K. Glazebrook, L. Hunt, T. Krühler, D. Le Borgne, M. Michałowski, E. Palazzi, A. Rau, A. Rossi, and P. Schady.

\section{References}

Baldry, I. K., Glazebrook, K., \& Driver, S. P., 2008, MNRAS, 388, 945

Bluck, A. F. L., Conselice, C. J., Buitrago, F., et al. 2012, ApJ, 747, 34

Gilbank, D. G., Bower, R. G., Glazebrook, K., et al., 2011, MNRAS , 414, 304

Hayes, M., Schaerer, D., \& Östlin, G. $2010 A \& A$, 509, L5

Heger, A., Fryer, C. L., Woosley, S. E., Langer, N., \& Hartmann, D. H. 2003 ApJ, 591, 288

Hunt, L., Palazzi, E., Rossi, A., et al. 2011, ApJ, 736, L36

Juneau, S., Glazebrook, K., Crampton, D., et al. 2005, ApJ, 619, L135

Krühler, T., Fynbo, J. P. U., Geier, S., et al., 2012, A\&SA, submitted, arXiv:1203.1919

Krühler, T., Greiner, J., Schady, P., et al. 2011, A\& $A$, 534, A108

Levesque, E. M., Kewley, L. J., Graham, J. F., \& Fruchter, A. S. 2010, ApJ, 712, L26

Mannucci, F., Cresci, G., Maiolino, R., Marconi, A., \& Gnerucci, A., 2010, MNRAS, 408, 2115

Niino, Y., Hashimoto, T., Aoki, K., et al. 2012, PASJ, submitted, arXiv:1204.0583

Perley, D. A., Modjaz, M., Morgan, A. N., et al. 2011, ApJ, submitted, arXiv:1112.3963

Pozzetti, L., Bolzonella, M., Zucca, E., et al., 2010, A\& A, 523, A13

Santini, P., Fontana, A., Grazian, A., et al., 2012, A\&A, 538, A33

Savaglio, S., Glazebrook, K., Le Borgne, D., et al., 2005, ApJ, 635, 260

Savaglio, S., Glazebrook, K., \& Le Borgne, D., 2009, ApJ, 691, 182

Savaglio, S., Rau, A., Greiner, J., et al. 2012, MNRAS, 420, 627

Sobral, D., Smail, I., Best, P. N., et al., 2012, MNRAS, submitted, arXiv:1202.3436

\section{Discussion}

PERLEY: Regarding the point about long-wavelength observations being essential at $z>1.5$, I certainly agree. In the past year we've been getting ground-based submillimeter/radio data on several dark GRB hosts and so far seeing very few bright ULIRG-like systems. Since it was mentioned in your abstract, I was wondering if you had any Hershel observations that might shed light on this question as well.

SAVAGLIO: We are in the process of receiving Herschel data for a sample of 13 GRB hosts. They are selected for being associated with dark GRBs. A few are already detected. We will study in detail the spectral energy distributions (SED) spanning roughly a factor of 1,000 in wavelength. The SED fitting will give stellar mass, bolometric luminosity, and star-formation rate at redshifts $z \leqslant 2$. 